\title{
Speech Enhancement using an Improved MMSE Estimator with Laplacian Prior
}

\author{
M. Rashidi-nejad \\ Speech Processing Research Lab \\ ECE Dept. \\ Yazd University \\ Yazd, Iran
}

\author{
H. R. Abutalebi \\ Speech Processing Research Lab \\ ECE Dept. \\ Yazd University \\ Yazd, Iran \& \\ Idiap Research Institute ${ }^{1}$ \\ Martigny, Switzerland
}

\author{
A. A. Tadaion \\ ECE Dept. \\ Yazd University \\ Yazd, Iran
}

\begin{abstract}
In this paper we present an optimal estimator of magnitude spectrum for speech enhancement when the clean speech DFT coefficients are modeled by a Laplacian distribution and the noise DFT coefficients are modeled by a Gaussian distribution. Chen has already introduced a Minimum Mean Square Error (MMSE) estimator of the magnitude spectrum. However, the proposed estimator, namely LapMMSE, does not have a closed form and is computationally extensive. We use his formulation for the MMSE estimator, employ some approximations and propose a computationally effective estimator for the magnitude spectrum. Experimental studies demonstrate better performance of our proposed estimator, Improved LapMMSE (ImpLapMMSE) Compared to LapMMSE and previous estimators in which Laplacian and Gaussian assumptions were made.
\end{abstract}

Keywords: MMSE estimator; Speech enhancement; Laplacian speech modeling; ImpLapMMSE

\section{INTRODUCTION}

In increasing number of speech processing applications, the noise reduction is becoming an essential pre-processing to improve the system performance. In the past three decades Minimum Mean Square Error (MMSE)-based singlechannel speech enhancement algorithms have received a lot of attention. In [1], Ephraim and Malah proposed a basic estimator for the magnitude spectrum of the speech signal. in [2], they introduced a log spectral amplitude (LSA) estimator and in [3], Cohen proposed optimally-modified log spectral amplitude (OM-LSA) estimator. Most of the previous works consider a fundamental assumption that the real and imaginary parts of Discrete Fourier Transform (DFT) coefficients of clean speech signal are modeled by Gaussian distribution. More recently, researchers have searched for adopting a statistical model for speech signal in order to enhance speech estimators. In this procedure, nonGaussian distributions have been employed to model real and imaginary parts of DFT coefficients of clean speech signals. Generally, Gamma or Laplacian distribution can be used to model real and imaginary part of clean DFT coefficients [4]-[9].

In [10], Chen and Loizou have proposed a MMSE estimator of the magnitude spectrum based on Laplacian model for speech probability density function (PDF). The proposed analytical solution is highly non-linear, computationally complex and very time-consuming for implementation. In [10], Chen showed that the magnitude and phase of the DFT coefficients are statistically independent. In this method, they approximated the probability density function of the DFT coefficient magnitude. This approximate is still computationally far-reaching. In this paper, we present some approximations for Bessel function as well as for the PDF of the magnitude spectrum of clean speech, $p_{X}(x)$, to reduce the complexity of the estimator. Simulation results demonstrate the complexity and performance of the resulting estimator. Evaluating the proposed estimator, namely Improved LapMMSE (ImpLapMMSE) shows that the proposed estimator outperforms the previous LapMMSE with lower complexity.

The remaining of the paper is organized as follows. In Section II, we explain the estimator proposed by Chen in [10]. In Section III, we derive Improved Laplacian-based MMSE estimator. In Section IV, we evaluate the performance of ImpLapMMSE estimator. Finally, Section V concludes the paper.

II. LAPLACIAN-BASED SHORT-TIME SPECTRAL AMPLITUDE ESTIMATOR

Suppose that $y(n)=x(n)+d(n)$, where $y(n), x(n)$ and $d(n)$ denote noisy signal, clean speech signal, and additive noise, respectively. Taking the Fourier Transform of $y(n)$, we get:

$$
Y\left(\omega_{k}\right)=X\left(\omega_{k}\right)+D\left(\omega_{k}\right)
$$

for $\omega_{k}=\frac{2 \pi k}{N}$ where $\mathrm{k}=0,1,2, \ldots, \mathrm{N}-1$, and $\mathrm{N}$ is the frame length. The above equation can be also expressed in the following form:

\footnotetext{
${ }^{1}$ H. R. Abutalebi has been on sabbatical at Idiap Research Institute during Fall 2010-Summer 2011.
} 


$$
Y_{k} e^{j \theta_{y}(k)}=X_{k} e^{j \theta_{x}(k)}+D_{k} e^{j \theta_{d}(k)}
$$

where $\left\{Y_{k}, X_{k}, D_{k}\right\}$ denote the corresponding magnitude spectra and $\left\{\theta_{y}(k), \theta_{x}(k), \theta_{d}(k)\right\}$ denote the corresponding phase spectra of the noisy, clean and noise signals, respectively.

According to the Ephraim and Malah basic work [1], the MMSE estimator of the magnitude spectrum $X_{k}$ is obtained as follows:

$$
\begin{aligned}
& \hat{X}_{k}=E\left\{X_{k} \mid Y\left(\omega_{k}\right)\right\}, \quad k=0,1,2, \ldots, N-1 \\
& =\frac{\int_{0}^{\infty} \int_{0}^{2 \pi} X_{k} p\left(Y\left(\omega_{k}\right) \mid X_{k}, \theta_{k}\right) p\left(X_{k}, \theta_{k}\right) d \theta_{k} d X_{k}}{\int_{0}^{\infty} \int_{0}^{2 \pi} p\left(Y\left(\omega_{k}\right) \mid X_{k}, \theta_{k}\right) p\left(X_{k}, \theta_{k}\right) d \theta_{k} d X_{k}}
\end{aligned}
$$

where $E\{$.$\} denotes the expectation operator, \theta_{k} \triangleq \theta_{x}(k)$ for convenience, $p\left(X_{k}, \theta_{k}\right)$ is the joint pdf of the magnitude and phase spectra, $\lambda_{d}(k)$ denotes the noise variance and $p\left(Y\left(\omega_{k}\right) \mid X_{k}, \theta_{k}\right)$ is given by [1]:

$$
\begin{aligned}
& p\left(Y\left(\omega_{k}\right) \mid X_{k}, \theta_{k}\right)=\frac{1}{\pi \lambda_{d}(k)} \exp \left\{\left(-\frac{1}{\lambda_{d}(k)} \mid Y\left(\omega_{k}\right)-\right.\right. \\
& \left.\left.X\left(\omega_{k}\right)\right|^{2}\right\}
\end{aligned}
$$

Following the procedure in [11], it is easy to show for a Laplacian distribution that $p\left(X_{k}, \theta_{k}\right)$ is given by [10]:

$$
p\left(X_{k}, \theta_{k}\right)=\frac{X_{k}}{2 \sqrt{\lambda_{x}(k)}} \exp \left[-\frac{X_{k}}{\sqrt{\lambda_{x}(k)}}\left(\left|\cos \theta_{k}\right|+\left|\sin \theta_{k}\right|\right)\right]
$$

Where $\lambda_{x}(k)$ is the variance of kth clean DFT coefficients. Substituting Eq. (4) and Eq. (5) into Eq. (3), [10] has reached the following form of estimator:
The above equation gives the Laplacian MMSE estimator of the spectral magnitude. We will refer to this estimator as the Laplacian MMSE estimator (or briefly, LapMMSE). To the knowledge of the authors, the closed form solution of this equation does not exist. In [10], by applying some approximations, Chen proposed alternative solutions and came up with significant results as follows [10]:

$$
\hat{X}_{\mathrm{k}}=\frac{A_{k}+B_{k}}{C_{k}+D_{k}}
$$

where

$$
A_{k}=\frac{\left(\frac{Y_{k}^{2}}{\gamma_{k}}\right)^{2}}{2} \sum_{m=0}^{\infty} \frac{\Gamma\left(m+\frac{3}{2}\right)}{m ! \Gamma(m+1)}\left(\frac{\gamma_{k}}{2 \xi_{k}^{2} Y_{k}^{2}}\right)^{m} \cdot F\left(-m,-m ; 1 ; 2 \xi_{k}^{2} Y_{k}^{2}\right)
$$

$$
\begin{gathered}
B_{k}=\frac{8}{\pi} \sum_{n=1}^{\infty} \frac{1}{n} \sin \left(\frac{\pi n}{4}\right) \frac{\left(\frac{2 \gamma_{k}}{Y_{k}^{2}}\right)^{n}\left(\frac{\gamma_{k}}{Y_{k}^{2}}\right)^{-\frac{n+3}{2}}}{2^{n+1} \Gamma(n+1)} \\
\quad \sum_{m=0}^{\infty} \frac{\Gamma\left(m+\frac{1}{2} n+\frac{3}{2}\right)}{m ! \Gamma(m+1)}\left(\frac{\gamma_{k}}{2 \xi_{k}^{2} Y_{k}^{2}}\right)^{m} \cdot F(-m,-m ; n \\
\left.\quad+1 ; 2 \xi_{k}^{2} Y_{k}^{2}\right) \\
C_{k}=\frac{Y_{k}^{2}}{2 \gamma_{k}} \sum_{m=0}^{\infty} \frac{1}{m !}\left(\frac{\gamma_{k}}{2 \xi_{k}^{2} Y_{k}^{2}}\right)^{m} \cdot F\left(-m,-m ; 1 ; 2 \xi_{k}^{2} Y_{k}^{2}\right)
\end{gathered}
$$

Let $\xi_{k} \triangleq \frac{\lambda_{x}}{\lambda_{d}}$ and $\gamma_{k} \triangleq \frac{Y_{k}^{2}}{\lambda_{d}}$ which denote the a priori and posteriori SNRs, respectively [1]. Substituting these two in (6), the estimator is re-written as follows [10]:

$$
=\frac{\int_{0}^{\infty} X_{k}^{2} \exp \left(-\frac{\gamma_{k} X_{k}^{2}}{Y_{k}^{2}}\right) \int_{0}^{2 \pi} \exp \left[\frac{2 X_{k} \gamma_{k} \cos \theta_{k}}{Y_{k}}-\frac{X_{k} \sqrt{\gamma_{k}}}{Y_{k} \sqrt{\xi_{k}}}\left(\left|\cos \theta_{k}\right|+\left|\sin \theta_{k}\right|\right)\right] d \theta_{k} d X_{k}}{\int_{0}^{\infty} X_{k} \exp \left(-\frac{\gamma_{k} X_{k}^{2}}{Y_{k}^{2}}\right) \int_{0}^{2 \pi} \exp \left[\frac{2 X_{k} \gamma_{k} \cos \theta_{k}}{Y_{k}}-\frac{X_{k} \sqrt{\gamma_{k}}}{Y_{k} \sqrt{\xi_{k}}}\left(\left|\cos \theta_{k}\right|+\left|\sin \theta_{k}\right|\right)\right] d \theta_{k} d X_{k}}
$$




$$
\begin{aligned}
& D_{k}=\frac{8}{\pi} \sum_{n=1}^{\infty} \frac{1}{n} \sin \left(\frac{\pi n}{4}\right) \frac{\left(\frac{2 \gamma_{k}}{Y_{k}^{2}}\right)^{n}\left(\frac{\gamma_{k}}{Y_{k}^{2}}\right)^{-\frac{n+2}{2}}}{2^{n+1} \Gamma(n+1)} \\
& . \sum_{m=0}^{\infty} \frac{\Gamma\left(m+\frac{1}{2} n+1\right)}{m ! \Gamma(m+1)}\left(\frac{\gamma_{k}}{2 \xi_{k}^{2} Y_{k}^{2}}\right)^{m} \cdot F(-m,-m ; n+ \\
& \left.1 ; 2 \xi_{k}^{2} Y_{k}^{2}\right)
\end{aligned}
$$

where $\Gamma($.$) is the gamma function and F(a, b ; c ; d)$ is the Gaussian hypergeometic function. As shown in (8-a)-(8-d), the derived LapMMSE estimator is highly nonlinear and computationally complex. In the section III, we propose some other approximations to be applied in equation (7) and present a computationally-feasible estimator.

\section{DERIVATION OF IMPLAP-MMSE}

Chen et al. have already shown that the magnitude and phase of the complex DFT coefficients of clean speech signals are statistically independent and derived $P_{X, \theta}(x, \theta)$ as follows [10]. Foe the simplicity of notation, the frequency bin index, $k$, has been dropped.

$$
\begin{aligned}
P_{X, \theta}(x, \theta) \approx \frac{x}{\pi \sigma^{2}} & {\left[\frac{\pi}{4} I_{0}\left(-\frac{\sqrt{2}}{\sigma} x\right)\right.} \\
& \left.+2 \sum_{k=1}^{\infty} \frac{1}{k} I_{k}\left(-\frac{\sqrt{2}}{\sigma} x\right) \sin \frac{\pi k}{4}\right] u(x)
\end{aligned}
$$

Substituting Eq. (9) into Eq. (3) results in the LapMMSE estimator of Eq. (8). However, Eq. (8) is still highly complex and nonlinear. To solve this problem, in the followings, we employ better approximates for $p_{\mathrm{X}}(x)$ and Bessel function to reach a computationally-efficient estimator for Laplacianbased MMSE estimator.

\section{A. Derivation of Approximate LapMMSE Estimator}

As it is mentioned in [10], $P_{X}(x)$ is given by:

$$
\begin{aligned}
& P_{X}(x)=\frac{2 x}{\sigma^{2}} \int_{0}^{\frac{\pi}{4}} \exp \left(\frac{-\sqrt{2} x \cos \theta}{\sigma}\right) d \theta= \\
& \frac{2 x}{\sigma^{2}} \int_{0}^{\frac{\pi}{4}} \exp (A \cos \theta) d \theta, \quad x \geq 0
\end{aligned}
$$

where $A=-\frac{\sqrt{2}}{\sigma}$, and $\sigma^{2}=\lambda_{x}$. Eq. (10) can be approximated using Tailor series expansion around $x=0$ as:

$$
\begin{aligned}
& P_{X}(x)=\frac{2 x}{\sigma^{2}} \int_{0}^{\frac{\pi}{4}} \exp (A x \cos \theta) d \theta= \\
& \frac{2 x}{\sigma^{2}} \int_{0}^{\frac{\pi}{4}} \sum_{\mathrm{n}=0}^{\infty} \frac{(A x \cos \theta)^{n}}{n !} d \theta= \\
& \frac{2 x}{\sigma^{2}} \sum_{\mathrm{n}=0}^{\infty} \frac{(\mathrm{Ax})^{\mathrm{n}}}{\mathrm{n} !} \int_{0}^{\frac{\pi}{4}}(\cos \theta)^{\mathrm{n}} \mathrm{d} \theta
\end{aligned}
$$

We approximate $P_{X}(x)$ as follows:

$$
P_{X}(x) \approx \frac{2 x}{\sigma^{2}} \exp \left(-\sqrt{\frac{2}{\lambda_{x}}} x\right)
$$

In Figs. 1-2 we compare the original $P_{X}(x)$ and the proposed approximation (Eq. (12)) for different values of variances $(\sigma)$. As shown in Figs. 1-2, this approximation has good accuracy and an error less than $0.3 \%$. The plots of $P_{X}(x)$ function and the derived approximation indicate that the obtained approximate can follow the function well.

Now, we employ this approximation of $p_{\mathrm{x}}(x)$ in the estimator. By substituting Eq. (12) into (7) we get:

$$
\begin{aligned}
& \hat{X}= \frac{\int_{0}^{\infty} x^{2} \exp \left(-\frac{1}{\lambda_{d}} x^{2}\right) \exp \left(-\sqrt{\frac{2}{\lambda_{x}}} x\right) \int_{0}^{2 \pi} \exp \left(\frac{2 Y x}{\lambda_{d}}\right) d x d \theta}{\int_{0}^{\infty} x \exp \left(-\frac{1}{\lambda_{d}} x^{2}\right) \exp \left(-\sqrt{\frac{2}{\lambda_{x}}} x\right) \int_{0}^{2 \pi} \exp \left(\frac{2 Y x}{\lambda_{d}}\right) d x d \theta} \\
&=\frac{\int_{0}^{\infty} x^{2} \exp \left(-\frac{1}{\lambda_{d}} x^{2}\right) \exp \left(-\sqrt{\left.\frac{2}{\lambda_{x}} x\right) I_{0}\left(\frac{2 Y x}{\lambda_{d}}\right) d x}\right.}{\int_{0}^{\infty} x \exp \left(-\frac{1}{\lambda_{d}} x^{2}\right) \exp \left(-\sqrt{\left.\frac{2}{\lambda_{x}} x\right) I_{0}\left(\frac{2 Y x}{\lambda_{d}}\right) d x}\right.}
\end{aligned}
$$

Since there is no closed form solution for this integrals (nominator and denominator of Eq. (13)), in the following, we introduce some approximation for Bessel function and propose a closed form estimator.

\section{B. The Approximation of Bessel Function}

In this section, we approximate Bessel function for two cases:

1. Bessel function approximation for small arguments

2. Bessel function approximation for large arguments

\section{Bessel function approximation for small arguments}

For small arguments of SNR, we approximate the Bessel function $I_{0}$ using the Tailor series expansion around $x=0$. As expressed in [12], we have:

$$
I_{0}(x ; K) \triangleq \sum_{k=0}^{K-1}\left(\frac{x}{2}\right)^{2 k} \frac{1}{(k !)^{2}}
$$

Substituting this expression into Eq. (13) gives: 


$$
\begin{aligned}
& \hat{X}=\frac{\int_{0}^{\infty} x^{2} \exp \left(-\frac{1}{\lambda_{x}} x^{2}\right) \exp \left(-\sqrt{\frac{2}{\lambda_{x}}} x\right) \sum_{k=0}^{K-1}\left(\frac{Y x}{\lambda_{d}}\right)^{2 k} \frac{1}{(k !)^{2}} d x}{\int_{0}^{\infty} x \exp \left(-\frac{1}{\lambda_{d}} x^{2}\right) \exp \left(-\sqrt{\frac{2}{\lambda_{x}}} x\right) \sum_{k=0}^{K-1}\left(\frac{Y x}{\lambda_{d}}\right)^{2 k} \frac{1}{(k !)^{2}} d x}= \\
& \frac{\sum_{k=0}^{K-1}\left(\frac{Y}{\lambda_{d}}\right)^{2 k} \frac{1}{(k !)^{2}} \int_{0}^{\infty} x^{2+2 k} \exp \left(-\frac{1}{\lambda_{x}} x^{2}\right) \exp \left(-\sqrt{\frac{2}{\lambda_{x}}} x\right) d x}{\sum_{k=0}^{K-1}\left(\frac{Y}{\lambda_{d}}\right)^{2 k} \frac{1}{(k !)^{2}} \int_{0}^{\infty} x^{1+2 k} \exp \left(-\frac{1}{\lambda_{d}} x^{2}\right) \exp \left(-\sqrt{\frac{2}{\lambda_{x}}} x\right) d x}= \\
& \frac{\sum_{k=0}^{K-1}\left(\frac{Y}{\lambda_{d}}\right)^{2 k} \frac{1}{(k !)^{2}} \int_{0}^{\infty} x^{2+2 k} \exp \left(-\frac{1}{\lambda_{d}} x^{2}-\sqrt{\frac{2}{\lambda_{x}}} x\right) d x}{\sum_{k=0}^{K-1}\left(\frac{Y}{\lambda_{d}}\right)^{2 k} \frac{1}{(k !)^{2}} \int_{0}^{\infty} x^{1+2 k} \exp \left(-\frac{1}{\lambda_{d}} x^{2}-\sqrt{\frac{2}{\lambda_{x}}} x\right) d x}
\end{aligned}
$$

Using [13, Thm.6.643.2] we drive:

$$
\begin{gathered}
\hat{X}=\frac{\sum_{k=0}^{K-1}\left(\frac{Y}{\lambda_{d}}\right)^{2 k} \frac{1}{(k !)^{2}}\left(2 \sqrt{\frac{2}{\lambda_{x}}}\right)^{-\frac{2 k+3}{2}} \Gamma(2 k+3) \exp \left(\frac{1}{4} \frac{\lambda_{d}}{\lambda_{x}}\right) D_{-(2 k+3)}(T)}{\sum_{k=0}^{K-1}\left(\frac{Y}{\lambda_{d}}\right)^{2 k} \frac{1}{(k !)^{2}}\left(2 \sqrt{\frac{2}{\lambda_{x}}}\right)^{-(k+1)} \Gamma(2 k+2) \exp \left(\frac{1}{4} \frac{\lambda_{d}}{\lambda_{x}}\right) D_{-(2 k+2)}(T)} \\
=\frac{1}{\sqrt{2 \gamma}} \frac{\sum_{k=0}^{K-1}\left(\frac{Y}{\lambda_{d}}\right)^{2 k} \frac{1}{(k !)^{2}}\left(2 \sqrt{\frac{2}{\lambda_{x}}}\right)^{-k} \Gamma(2 k+3) \exp \left(\frac{1}{4} \frac{\lambda_{d}}{\lambda_{x}}\right) D_{-(2 k+3)}(T)}{\sum_{k=0}^{K-1}\left(\frac{Y}{\lambda_{d}}\right)^{2 k} \frac{1}{(k !)^{2}}\left(2 \sqrt{\frac{2}{\lambda_{x}}}\right)^{-k} \Gamma(2 k+2) \exp \left(\frac{1}{4} \frac{\lambda_{d}}{\lambda_{x}}\right) D_{-(2 k+2)}(T)} . Y
\end{gathered}
$$

By considering the a priori and a posteriori SNRs as $\xi=\frac{\lambda_{x}}{\lambda_{d}}$ and $\gamma=\frac{Y^{2}}{\lambda_{d}}$ respectively, equation (16) can b re-written as:
Substituting this approximation into Eq. (13) we get:

$\hat{X}$

$$
\begin{aligned}
= & \frac{\int_{0}^{\infty} x^{2} \exp \left(-\frac{1}{\lambda_{d}} x^{2}\right) \exp \left(-\sqrt{\frac{2}{\lambda_{x}}} x\right) \frac{1}{\sqrt{2 \pi x}} \exp \left(\frac{2 Y x}{\lambda_{d}}\right) d x}{\int_{0}^{\infty} x \exp \left(-\frac{1}{\lambda_{d}} x^{2}\right) \exp \left(-\sqrt{\frac{2}{\lambda_{x}}} x\right) \frac{1}{\sqrt{2 \pi x}} \exp \left(\frac{2 Y x}{\lambda_{d}}\right) d x} \\
= & \frac{\int_{0}^{\infty} x^{\frac{3}{2}} \exp \left(-\frac{1}{\lambda_{d}} x^{2}\right) \exp \left(-\sqrt{\frac{2}{\lambda_{x}}} x\right) \exp \left(\frac{2 Y x}{\lambda_{d}}\right) d x}{\int_{0}^{\infty} x^{\frac{1}{2}} \exp \left(-\frac{1}{\lambda_{d}} x^{2}\right) \exp \left(-\sqrt{\frac{2}{\lambda_{x}}} x\right) \exp \left(\frac{2 Y x}{\lambda_{d}}\right) d x} \\
= & \frac{\int_{0}^{\infty} x^{\frac{3}{2}} \exp \left(-\frac{1}{\lambda_{d}} x^{2}-\left(\sqrt{\frac{2}{\lambda_{x}}}-\frac{2 Y x}{\lambda_{d}}\right) x\right) d x}{\int_{0}^{\infty} x^{\frac{1}{2}} \exp \left(-\frac{1}{\lambda_{d}} x^{2}-\left(\sqrt{\frac{2}{\lambda_{x}}}-\frac{2 Y x}{\lambda_{d}}\right) x\right) d x}
\end{aligned}
$$

Using [13, Thm 3.426.1] we get:

$$
\begin{aligned}
& \hat{X}=\frac{1}{\sqrt{2 \gamma}} \frac{\Gamma\left(\frac{5}{2}\right) \exp \left(\frac{P^{2}}{4}\right) D_{-\frac{5}{2}}(P)}{\Gamma\left(\frac{3}{2}\right) \exp \left(\frac{P^{2}}{4}\right) D_{-\frac{3}{2}}(P)} Y \\
& =\frac{1}{\sqrt{2 \gamma}} \frac{\Gamma\left(\frac{5}{2}\right) D_{-\frac{5}{2}}(P)}{\Gamma\left(\frac{3}{2}\right) D_{-\frac{3}{2}}(P)} Y
\end{aligned}
$$

where $P=\left(\sqrt{\frac{1}{\xi}}-\sqrt{2 \gamma}\right)$ and $D_{\gamma}(z)$ denote parabolic cylinder function. Therefore defining $G_{\text {ImPlap-MMSE }}$ as follows:

$\hat{X}$

$=\frac{1}{\sqrt{2 \gamma}} \frac{\sum_{k=0}^{K-1}\left(\frac{Y}{\lambda_{d}}\right)^{2 k} \frac{1}{(k !)^{2}}\left(\frac{2}{Y} \sqrt{\frac{\gamma}{\xi}}\right)^{-k} \Gamma(2 k+3) \exp \left(\frac{1}{4 \xi}\right) D_{-(2 k+3)}(T)}{\sum_{k=0}^{K-1}\left(\frac{Y}{\lambda_{d}}\right)^{2 k} \frac{1}{(k !)^{2}}\left(\frac{2}{Y} \sqrt{\frac{\gamma}{\xi}}\right)^{-k} \Gamma(2 k+2) \exp \left(\frac{1}{4 \xi}\right) D_{-(2 k+2)}(T)} . Y$

Where $T=\sqrt{\gamma}$ and $D_{\gamma}(z)$ is parabolic cylinder function.

\section{Bessel function approximation for large arguments}

For large input arguments, a large value of $\mathrm{K}$ is required to accurately approximate $I_{0}$ via equation (14). This results in numerical problems and demanding computations. Therefore, for these case the following well-known approximation of $I_{0}$ is applied [12]:

$$
I_{0}(x) \sim \frac{1}{\sqrt{2 \pi x}} \exp (x)
$$

$$
G_{I m p L a p-M M S E}=\frac{1}{\sqrt{2 \gamma}} \frac{\Gamma\left(\frac{5}{2}\right) D_{-\frac{5}{2}}(P)}{\Gamma\left(\frac{3}{2}\right) D_{-\frac{3}{2}}(P)}
$$

We get:

$$
\widehat{X}=G_{\text {ImpLap }-M M S E} \cdot Y
$$

Finally, the clean speech component is obtained using the inverse STFT and the weighted overlap-add method. 


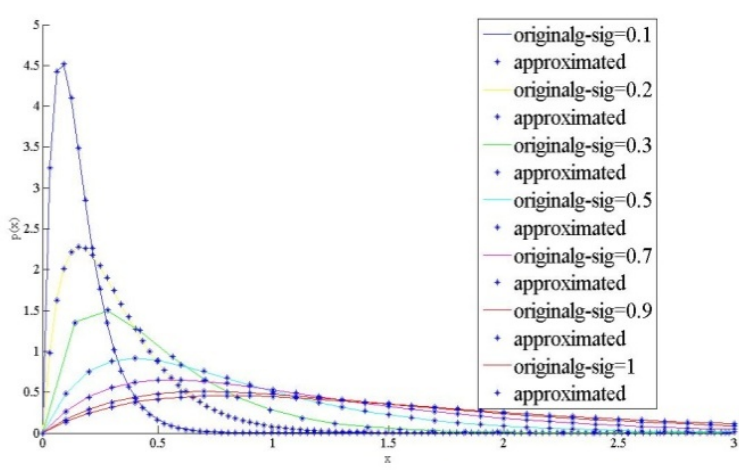

(a)

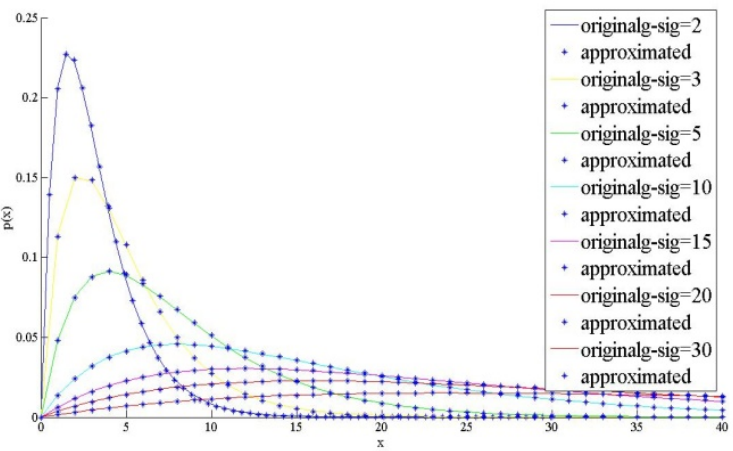

(b)

Fig. 1. The pdf of the magnitude of the DFT coefficients assuming the real and imaginary parts are modeled by a Laplacian distribution for various $\sigma$ The plot indicated by the solid line shows the pdf computed by numerical integration of Eq. (10). The plot indicated by '*' shows the pdf approximated by Eq. (12). (a) $\sigma=0.1,0.2,0.3,0.5,0.7,0.9,1$. (b) $\sigma=$ $2,3,5,10,15,20,30$.

\section{IMPLEMENTATION AND PERFORMANCE EVALUATION}

In this section, we present experimental results that demonstrate better performance of our proposed estimator, Improved LapMMSE (ImpLapMMSE) with respect to LapMMSE and previous estimators in which Laplacian and Gaussian assumptions were made. For simulation, eight (clean) speech signals (sampled at $16 \mathrm{KHz}$ ) were selected from TIMIT data base. Further. We made these signals noisy with Gaussian noise and considered wide range of input SNRs $(-10 \mathrm{db},-7 \mathrm{db},-2.5 \mathrm{db}, 0 \mathrm{db})$ in this experiment. To evaluate the performance of proposed method, we have used three basic measures: SegSNR, LLR distance and Weighted Spectral Slope WSS. Next, we have compared the output of proposed method with those for MMSE-STSA, MMSELSA and OM-LSA, methods.

The results have been listed in Tables I-III for segSNR, LLR distance and WSS values, respectively. As shown in simulation results, we realize that the proposed method in lower SNR values has gained significant performance, In comparison to the amplitude estimation of signal spectrum when Gaussian assumption was considered. Furthermore, the proposed estimator provides better performance than MMSE, LSA and OMLSA methods. Listening tests show that this method has less residual noise than the previous estimators. This is also almost obvious in Fig. 2

\section{SUMMARY AND CONCLUSION}

In this paper, we explored the Laplacian MMSE estimator (proposed by Chen et al. in [10]). The estimator is based on strongly analytical mathematics, and it has a great complexity. In this paper, we employed more accurate approximations to improve MMSE estimator in which DFT coefficients of speech signal are modeled by Laplasian distribution. As a result we obtained a Gain function which can omit the additive noise in a better way. Comparing the proposed method with the previous ones shows this fact and as in [10], this method has less residual noise, less distortion speech signal and finally better performance in results.

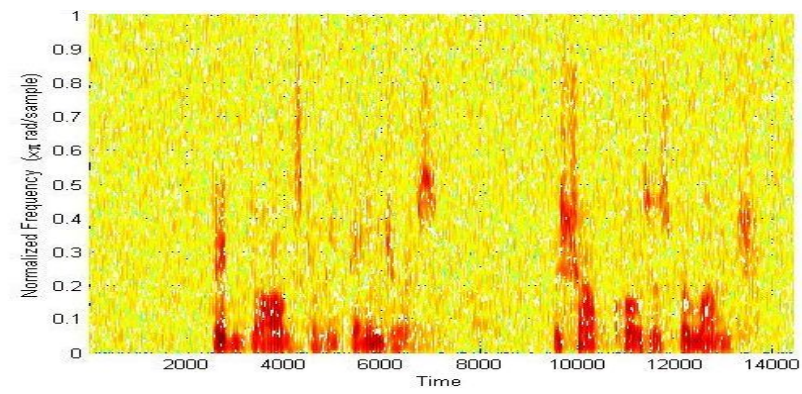

(a)

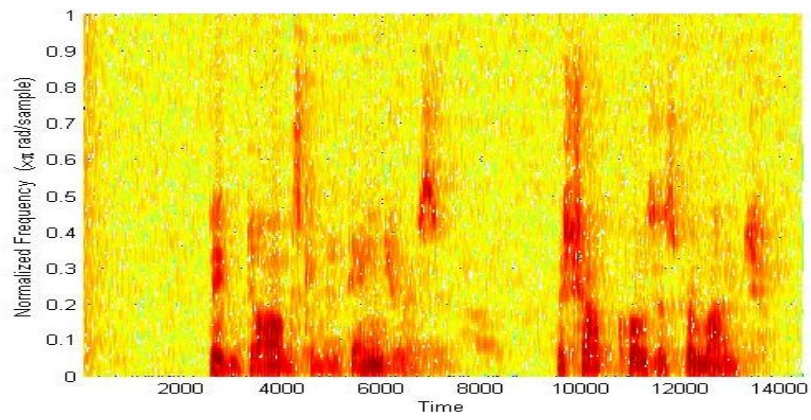

(b)

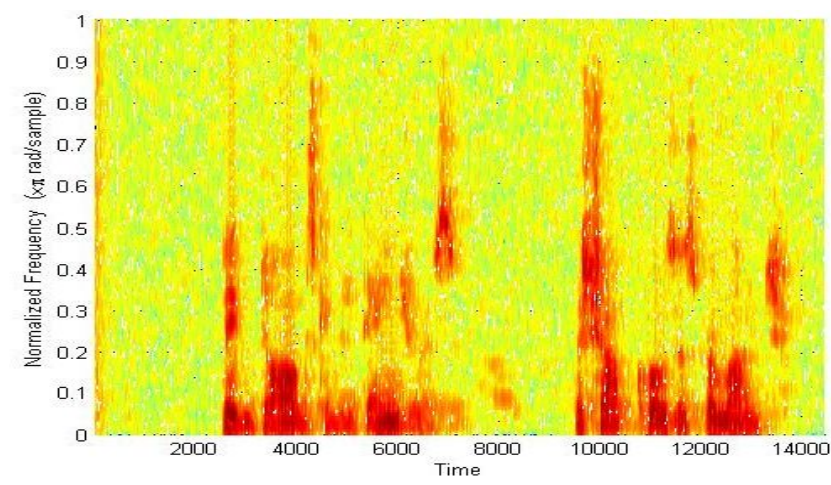

(c) 


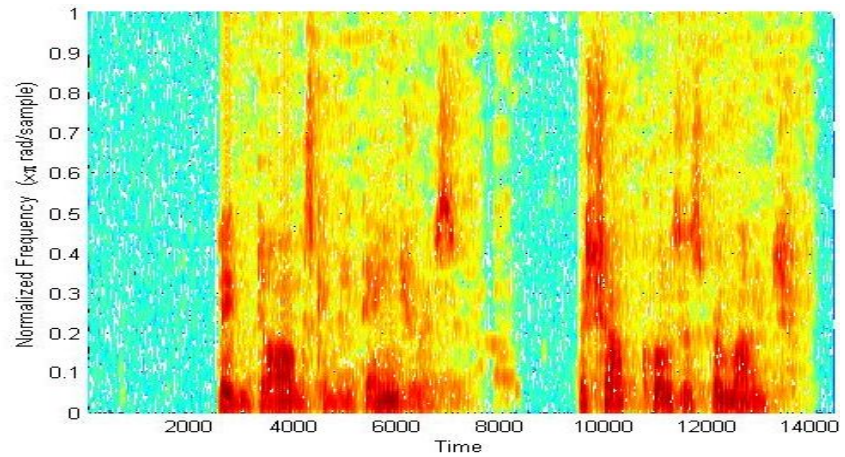

(d)

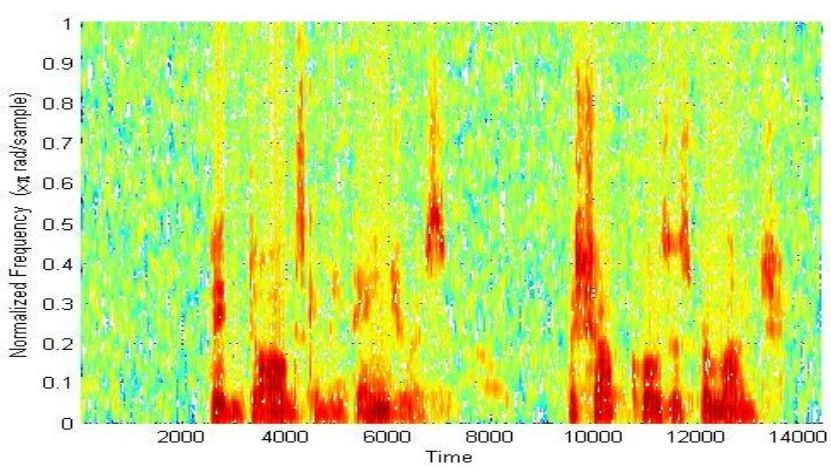

(e)

Fig. 2. Spectrograms of (a) Noisy signals, (b) enhancement noisy signal by the Gaussian MMSE estimator, (c) enhancement noisy signal by the Gaussian MMSE-LSA estimator (d) enhancement noisy signal by the Gaussian MMSE-OMLSA estimator (e) enhancement noisy signal by the Laplacian MMSE (ImpLapMMSE) estimator

\section{TABALE I}

Comparative performance, in terms of segmental SNR, of the Gaussianbased MMSE, MMSE_LSA, MMSE-OMLSA and ImpLaplacian-based MMSE estimators

\begin{tabular}{|c|c|c|c|c|c|}
\cline { 2 - 6 } \multicolumn{1}{c|}{} & \multicolumn{5}{c|}{ Segmental SNR (dB) } \\
\hline Method & $-10 \mathrm{~dB}$ & $-7 \mathrm{~dB}$ & $-5 \mathrm{~dB}$ & $-2.5 \mathrm{~dB}$ & $0 \mathrm{~dB}$ \\
\hline ImpLapMMSE & 2.42 & 3.84 & 5.22 & 7.68 & 11.20 \\
\hline MMSE-STSA & 1.40 & 2.55 & 3.62 & 3.23 & 8.32 \\
\hline MMSE-LSA & 1.94 & 3.30 & 4.50 & 6.60 & 9.57 \\
\hline MMSE-OMLSA & 3.06 & 4.61 & 6.08 & 8.61 & 12.27 \\
\hline
\end{tabular}

TABALE II

Comparative performance, in terms of log-likelihood ratio, of the Gaussianbased MMSE, MMSE LSA, MMSE-OMLSA and ImpLaplacian-based MMSE estimators

\begin{tabular}{|c|c|c|c|c|c|}
\cline { 2 - 6 } \multicolumn{1}{c|}{} & \multicolumn{5}{c|}{ log-likelihood ratio (LLR) (dB) } \\
\hline Method & $-10 \mathrm{~dB}$ & $-7 \mathrm{~dB}$ & $-5 \mathrm{~dB}$ & $-2.5 \mathrm{~dB}$ & $0 \mathrm{~dB}$ \\
\hline ImpLapMMSE & 3.44 & 3.21 & 3.05 & 2.88 & 2.74 \\
\hline MMSE-STSA & 3.40 & 3.16 & 3.01 & 2.83 & 2.67 \\
\hline MMSE-LSA & 3.33 & 3.10 & 2.94 & 2.75 & 2.61 \\
\hline MMSE-OMLSA & 3.16 & 3.94 & 2.78 & 2.61 & 2.49 \\
\hline
\end{tabular}

TABALE III

Comparative performance, in terms of WSS of the Gaussian-based MMSE, MMSE_LSA, MMSE-OMLSA and ImpLaplacian-based MMSE estimators

\begin{tabular}{|c|c|c|c|c|c|}
\cline { 2 - 6 } \multicolumn{1}{c|}{} & \multicolumn{5}{c|}{ Weighted Spectral Slope (WSS) (dB) } \\
\hline Method & $-10 \mathrm{~dB}$ & $-7 \mathrm{~dB}$ & $-5 \mathrm{~dB}$ & $-2.5 \mathrm{~dB}$ & $0 \mathrm{~dB}$ \\
\hline ImpLapMMSE & 133.41 & 128.49 & 125.50 & 120.33 & 115.33 \\
\hline MMSE-STSA & 64.40 & 62.47 & 61.04 & 59.16 & 57.25 \\
\hline LSA & 68.51 & 66.46 & 65.13 & 63.29 & 61.15 \\
\hline OM-LSA & 90.12 & 91.74 & 92.51 & 92.36 & 90.19 \\
\hline
\end{tabular}

\section{REFERENCES}

[1] Y. Ephraim and D. Malah, "Speech enhancement using a minimum mean square error short-time spectral amplitude estimator," IEEE Trans. Acoust, Speech, Signal Process, vol. ASSP-32, no. 6, pp. 1109-1121, Dec 1984.

[2] Y. Ephraim, D. Malah, "Speech enhancement using a minimum mean square error log-spectral amplitude estimator", IEEE Trans. Acoust. Speech Signal Process. April 1985.

[3] I. Cohen, "On speech enhancement under signal presence uncertainty," Proceedings of the 26th IEEE International Conference on Acoustics Speech, and Signal Processing, ICASSP-01, May 2001.

[4] R. Martin, "Speech enhancement using MMSE short time spectral estimation with gamma distributed priors,". In: Proc. IEEE ICASSP. pp.504-512, 2002.

[5] R. Martin, C. Breithaupt, "Speech enhancement in the DFT domain using Laplacian speech priors,". In Proc. 8th Intern. Workshop on Acoustic Echo and Noise Control (IWAENC), vol. 8-11. Kyoto, Japan, pp. 87-90, 2003.

[6] T. Lotter, P Vary, "Noise reduction by maximum a posteriori spectral amplitude estimation with Supergaussian speech modeling,". In proc. Intern. Workshop Acoust. Echo Noise Control, Kyoto, Japan, 2003.

[7] C. Breithaupt, R. Martin, "MMSE estimation of magnitudesquared DFT coefficients with SuperGaussian priors,". In: Proc. IEEE ICASSP, vol. I, pp. 896-899. 2003.

[8] J. Porter, S. Boll, "Optimal estimators for spectral restoration of noisy speech,”. In: Proc. IEEE ICASSP. pp. 18A.2.1-18A.2.4. 1984.

[9] B. Chen, P. Loizou, "Speech enhancement using a MMSE short time spectral amplitude estimator with Laplacian speech modeling,". In: Proc. IEEE ICASSP, vol. 1, pp. 1097-1100. 2005.

[10] B. Chen, C. Loizou, "A Laplacian-based MMSE estimator for speech enhancement," Speech Communication vol.49 pp. 134-143. 2007.

[11] A. Papoulis, U. Pillai ,Probability, Random Variables and Stochastic Processes, Fourth ed., McGraw-Hill. 2001.

[12] M. Abramowitz and I. A. Stegun, Handbook of Mathematical Functions, Dover, New-York, 9th Dover printing. 1964.

[13] I. Gradshteyn and I. Ryzhik, Table of Integrals, Series and Products, New York: Academic, 6th edition, 2000.

[14] R.C. Hendriks, J.S. Erkelens, J. Jensen, and R. Heusdens, "Minimum Mean-Square Error Amplitude estimator for speech enhancement under the generalized gamma distribution," in proc. of IWARNC. 2006 - Paris - September 12-14, 2006. 